\title{
A vendor-buyer coordinated system featuring an unreliable machine, scrap, outsourcing, and multiple shipments
}

\author{
Yuan-Shyi Peter Chiu ${ }^{a}$, Zhong-Yun Zhao ${ }^{a}$, Singa Wang Chiu ${ }^{b^{*}}$ and Victoria Chiu ${ }^{\mathrm{c}}$
}

a Department of Industrial Engineering and Management, Chaoyang University of Technology, Taichung 413, Taiwan

${ }^{b}$ Department of Business Administration, Chaoyang University of Technology, Taichung 413, Taiwan

${ }^{c}$ Department of Accounting, Finance and Law, State University of New York at Oswego, Oswego, NY 13126, USA

\begin{abstract}
A B S T R A C T
Operating in today's highly competitive global markets, transnational enterprises always seek to optimize internal vendor-buyer coordinated systems to ensure timeliness and quality deliveries, given the reality of unreliable machines and limited capacity. To facilitate accurate decision making to help organizations gain competitive advantages in such situations, this study explores an intra-supply-chain problem featuring a partial outsourcing batch fabrication plan, random scrap, Poisson-distributed breakdown rate, and multiple shipments of end-product. First, we build a model to characterize the problem clearly. Then, we carry out formulations, analyses, and derivations of the model to attain the problem's cost function. We then use differential calculus and propose a specific algorithm to confirm the convexity of the obtained cost function and derive the optimal runtime. Finally, we offer a numerical illustration to demonstrate the result's applicability for other business circumstances. Additional elements of the problem are then discussed, including the individual and combined influence of variations in scrap, outsourcing, breakdown, and shipping frequency. The features of an optimal operating policy and cost relevant parameters are now revealed to assist management with strategic planning and decision making in real-world intra-supply-chain environments.
\end{abstract}

\section{Introduction}

Transnational firms, operate in today's highly competitive world markets, constantly pursue to optimize internal vendor-buyer coordinated systems to ensure timeliness and quality deliveries, given the reality of unreliable machines and limited capacity. To facilitate accurate decision making to help organizations gain competitive advantages in such situations, this study explores an intra-supply-chain problem featuring a partial outsourcing batch fabrication plan, random scrap, Poisson-distributed breakdown rate, and multiple shipments of end-product. Unreliable production equipment is a troubling issue in most real manufacturing environments and it interrupts fabrication process and hence, draws special attentions of operation management. Alam and Sarma (1974) studied a deteriorating equipment which is subject to breakdown and determined its optimal maintenance schedule. Chakravarthy (1983) analyzed the parallel system's reliability, wherein multiple identical components in the system are subject to exponential failures and have the phase type repair times. Alvarez-Vergas et al. (1994) considered the continuousflow production lines with finite buffers and unreliable machines. Seventy manufacturing lines were

\footnotetext{
* Corresponding author

E-mail: swang@.cyut.edu.tw (S.W. Chiu)

2020 Growing Science Ltd.

doi: $10.5267 /$ j.ijiec.2020.1.004
} 
simulated with various production rates and other performance indexes to demonstrate that it is a decent approximation to the asynchronous model. Levitin (2003) considered the linear multi-state elements allocation problem with vulnerable nodes. The connected nodes of elements can be ruined by a probabilistic external impact, and when both internal failures and external impact take place, the system can still survive if at least one good connected path exists from the source to the sink. The author proposed a genetic optimization-tool algorithm and an algorithm for seeking the multi-state elements distribution. Golmakani and Moakedi (2012) studied an unreliable system with two repairable components. When the first component fails (only detected through inspection), the operating cost increases, and it has no impact on the second component. Conversely, when the second component breaks, the first component's failure rate increases. A periodic inspection is implemented on the first component, and the authors proposed a model to seek the optimal inspection schedule that keeps the total cost at minimum. Chiu et al. (2019a) explored the joint influences of backorder, random failures, scrap, and rework on the inventory replenishing decision. The authors first built a model to characterize the problem and then carried out formulations, analyses, and derivations of the model to attain the cost function. The differential calculus and a specific algorithm were utilized to confirm the convexity of the cost function and derive the optimal runtime. A numerical illustration was offered to show their result's applicability. Additional studies (Köksal et al., 2013; Shakoor et al., 2017; Souha et al., 2018; Zahraee et al., 2018; Lin et al., 2019) examined the impact of random defective/scrap rate and different characteristics of unreliable equipment on the manufacturing and operations management.

To smooth the manufacturing schedules and/or shorten manufacturing runtime, an effective option used by the production managers is to outsource a portion of a lot. Kamien and Li (1990) proposed a model to explore an aggregate planning strategy incorporating flexibility, subcontracting, production smoothing, and coordination. Different ways of subcontracting and their relevant expenses were discussed, with the aim of identifying potential feasible outsourcing mechanisms in coordinating inhouse fabrication and outside providers. Bryce and Useem (1998) evaluated the influence of outsourcing strategy on the corporation's value, with the purpose of investigating the real influence of outsourcing strategy on the growing markets and what will be outsourcing's long-term perspectives in the future. The authors also pointed out with evidence, the benefits of outsourcing when it is well designed and managed. Lee and Sung (2008) explored a scheduling problem incorporating an outsourcing option, wherein, any job is either processed in-house on a single machine or by the outside provider, with the purpose of minimizing total completion times under the outsourcing budget constraint. Due the NP-hard nature of the problem, the authors proposed heuristics and branch-and-bound algorithms to help characterize properties to the solution of the problem. Swenseth and Olson (2016) studied the trade-offs of lean systems versus outsourced strategies in supply chain environments. The authors evaluated lean systems' cost impacts versus the advantage of purchasing cost in global supply-chain, the performance of the latter was measured through simulation that focused on the impact of inventory factors and potential profit. The results indicated that in certain conditions the lower procuring cost may override lean systems' shortterm stock holding cost savings. Other studies (Çınar \& Güllü, 2012; Chiu et al., 2017; Mohammadi, 2017; Chiu et al., 2019b) also explored diverse features of outsourcing strategies effect on company's fabrication systems and overall operations.

Unlike a continuous stock issuing policy assumed by the conventional economical batch size model (Taft, 1918), the end-product delivery policy in real-world supply chains is multiple shipments at fixed time intervals. Hill (1996) studied a finite-rate fabrication system with the raw material purchase, manufacturing, and shipment of fixed-quantity end-item at client requested time intervals. The author successfully decided the cost-minimization purchasing and manufacturing schedule. Siajadi et al. (2006) considered a multi-buyer single-vendor fabrication-transportation problem, with the aim of minimizing the joint total system related cost for both parties. The authors proposed a method to first examine a single-vendor two-buyer model, and then extended to consider the model with multiple buyers, the exact optimal solution and an approximate optimal solution were gained, respectively. Sarker (2013) developed 
fabrication-inventory models to explore the probabilistic deterioration item in the two-echelon supplychain environments. Three distinct continuous probability distributions for deterioration were examined to jointly decide the optimal batch-size and frequency of shipments that minimize total costs. Numerical illustrations were offered to show the difference among three models and their applicability. Other studies (Kuhn and Liske, 2011; Stažnik et al., 2017; Díaz-Mateus et al., 2018; Morales et al., 2018; Rahimi and Fazlollahtabar, 2018; Al-Odeh and Altarazi, 2019; Mosca et al., 2019) also examined different features of multi-shipment effect on various fabrication-transportation and supply-chain systems. Few studies have investigated the joint influences of unreliable machine, scrap, outsourcing, and multiple shipments on the intra-supply-chain planning, this study aims to fill the gap.

\section{Problem description and modelling}

\subsection{Nomenclature}

$Q$ = replenishing lot-size,

$T^{\prime} \pi=$ cycle time in the breakdown happening case of the proposed system,

$t_{1 \pi}=$ replenishing uptime in the proposed system - the decision variable,

$\pi=$ the outsourcing portion of a batch in each cycle (where $0<\pi<1$ ),

$K=$ the in-house manufacturing setup cost,

$C=$ the in-house manufacturing unit cost,

$K_{\pi}=$ the outsourcing setup cost (where $\left.K_{\pi}=\left(1+\beta_{1}\right) K\right)$,

$C_{\pi}=$ the outsourcing unit cost (assuming $C_{\pi}=\left(1+\beta_{2}\right) C$ ),

$\beta_{1}=$ connecting parameter between $K_{\pi}$ and $K$ (where $-1<\beta_{1}<0$ ),

$\beta_{2}=$ connecting parameter between $C_{\pi}$ and $C$ (where $\beta_{2}>0$ ),

$h$ = unit holding cost,

$h_{2}=$ unit holding cost at buyer end,

$C_{\mathrm{S}}=$ unit disposal cost,

$C_{1}=$ unit cost for safety item,

$h_{3}=$ unit holding cost for safety item,

$t=$ time to a breakdown happening - it obeys the Exponential distribution,

$f(t)=$ the density function of $t$ (where $\left.f(t)=\beta e^{-\beta t}\right)$,

$\mathrm{F}(t)=$ the cumulative density function of $t$ (where $\left.\mathrm{F}(t)=\left(1-e^{-\beta t}\right)\right)$,

$M=$ repair cost per breakdown,

$\beta=$ the mean Poisson distributed breakdown rate (in a year),

$t_{\mathrm{r}}=$ the breakdown repair time,

$P_{1}=$ in-house annual fabrication rate (where $d_{1}=P_{1 x}$ ),

$x=$ random scrap portion a batch in each cycle (where $0<x<1$ ),

$d_{1}=$ fabrication rate of scraps (where $d_{1}=P_{1} x$ ),

$t^{\prime} 2 \pi=$ distribution time of finished products,

$n=$ number of shipments in a cycle,

$t_{\mathrm{n} \pi}^{\prime}=$ time interval between shipments (where $\left.t_{\mathrm{n} \pi}^{\prime}=t^{\prime} 2 \pi / n\right)$,

$C_{\mathrm{T}}=$ unit transportation cost,

$K_{1}=$ fixed transportation cost,

$H_{0}=$ finished stock level when a breakdown occurs,

$H_{1}=$ finished stock level when uptime ends,

$H$ = finished stock level when outsourced items are received,

$g=t_{\mathrm{r}}$,

$D$ = quantity per shipment,

$I=$ the leftover products in each $t^{\prime} \mathrm{n} \pi$,

$I(t)=$ finished stock level at time $t$,

$I_{\mathrm{F}}(t)=$ safety stock level at time $t$,

$I_{\mathrm{s}}(t)=$ scrapped stock level at time $t$, 
$I_{\mathrm{c}}(t)=$ buyer stock level at time $t$,

$T C\left(t_{1 \pi}\right)_{1}=$ total system cost per cycle in the breakdown happening case,

$E\left[T C\left(t_{1 \pi}\right)_{1}\right]=$ expected total system cost per cycle in the breakdown happening case,

$E\left[T^{\prime} \pi\right]=$ the expected cycle time in the breakdown happening case,

$t_{2 \pi}=$ distribution time of finished products in the no breakdown case,

$t_{\mathrm{n} \pi}=$ time interval between shipments in the case that no breakdown happens,

$T_{\pi}=$ cycle time in the case that no breakdown happens,

$T C\left(t_{1 \pi}\right)_{2}=$ total system cost per cycle in the no breakdown case,

$\mathrm{E}\left[T C\left(t_{1 \pi}\right)_{2}\right]=$ the expected total system cost per cycle in the no breakdown case,

$\mathrm{E}\left[T C U\left(t_{1 \pi}\right)\right]=$ expected annual system cost in the no breakdown case,

$E\left[T_{\pi}\right]=$ the expected cycle time in the no breakdown case,

$t_{1}=$ uptime of the proposed system without outsourcing, nor breakdown,

$t_{2}=$ distribution time of the proposed system without outsourcing, nor breakdown,

$T$ = cycle time of the proposed system without outsourcing, nor breakdown,

$T_{\pi}=$ cycle time of the proposed system with or without breakdown happening,

\subsection{Problem description}

This study explores a vendor-buyer coordinated system featuring unreliable machine, random scrap, outsourcing, and multi-shipment distribution plan. Consider that a buyer routinely purchases $\lambda$ units of a particular product per year from a vendor, and a batch fabrication along with a multi-shipment policy is used by the vendor to meet the requirements. The vendor's annual fabrication rate is $P_{1}$ and lot size is $Q$. However, to reduce the batch cycle/response time, a $\pi$ portion of $Q$ is provided by an external contractor, who guarantees the quality of outsourced items and promises its receipt schedule, which is on the beginning of vendor's distribution time of finished items (i.e., $t^{\prime} 2 \pi$ ). Thus, different setup and unit costs, $K_{\pi}$ and $C_{\pi}$ are associated with this specific outsourcing option (refer to Nomenclature for their relationships with in-house relevant costs). During the fabrication of remaining lot (i.e., $(1-\pi) Q)$, the machine is not reliable, it randomly produces $x$ portion of scrap at a rate $d_{1}$ (hence, $d_{1}=x P_{1}$ ), and it is also subject to a Poisson distributed breakdown with mean rate $\beta$ per year. All scraps are disposed at an extra unit cost $C_{\mathrm{s}}$. Once a breakdown takes place, machine is under repair at once, and the incomplete lot will be resumed immediately once the machine is restored. The cost for machine repair is $M$, and a constant repair time $t_{\mathrm{r}}$ is assumed; in case that actual repair time shall exceed $t_{\mathrm{r}}$, a rental machine will be put in use to avoid further delay in fabrication. Upon completion of the uptime and receipt of outsourced stock, $n$ equal-size fixed amount of the lot are distributed to the buyer at fixed time interval $t^{\prime}{ }_{n \pi}$, then, the next fabrication cycle starts. Shortage situation is not allowed in this study, so $\left(P_{1}-d_{1}-\lambda\right)$ must be $>0$.

\subsection{Modelling}

According to the Poisson distributed breakdown rate, two distinct conditions need to be separately studied, as follows:

\subsubsection{Condition 1: A Poisson breakdown happens during $t_{1 \pi}$}

In condition one, the time to a breakdown happening $t<t_{1 \pi}$. Fig. 1 illustrates the finished stock level in the proposed system considering random scrap, outsourcing, stochastic breakdown, and multi-shipment distribution plan. 


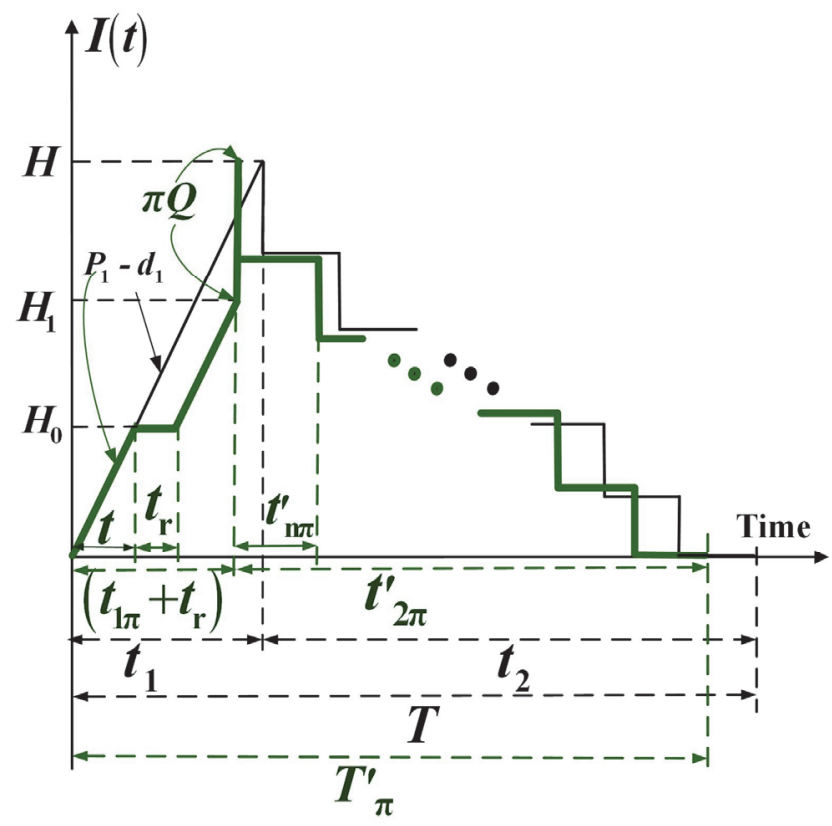

Fig. 1. The finished stock level in the proposed system considering random scrap, outsourcing, stochastic breakdown, and multi-shipment distribution plan (in green) as compared to that of a batch system with scrap and multi-shipment plan (in black)

Fig. 1 depicts that the finished stock arrives at $H_{0}$ at the time a breakdown happens, and once the breakdown is repaired, the finished stock continues to pile up and reach $H_{1}$ when replenishing uptime ends. Then, in the beginning of the distribution time $t^{\prime} 2 \pi$, the outsourced products are received, and also due to a breakdown occurrence, the safety stock $\lambda t_{\mathrm{r}}$ is also required for meeting the demand in $t_{\mathrm{r}}$ (see Fig. 2). Hence, prior to the distribution time, total finished stocks go up to $H$ (see Eqs. (1-3) for details).

$$
\begin{aligned}
& H_{0}=\left(P_{1}-d_{1}\right) t \\
& H_{1}=\left(P_{1}-d_{1}\right) t_{1 \pi} \\
& H=H_{1}+\pi Q+\lambda t_{r}
\end{aligned}
$$

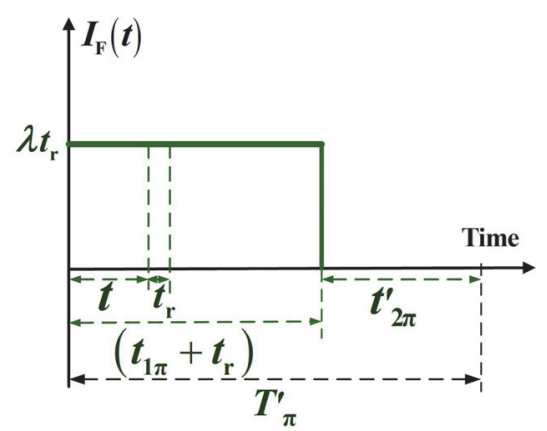

Fig. 2. The safety stock level in condition 1 of the proposed system

The following formulas can also be directly observed from Fig. 1:

$$
\begin{aligned}
& T^{\prime}{ }_{\pi}=t_{1 \pi}+t_{\mathrm{r}}+t^{\prime}{ }_{2 \pi} \\
& t_{1 \pi}=\frac{Q(1-\pi)}{P_{1}} \\
& t^{\prime}{ }_{2 \pi}=T^{\prime}{ }_{\pi}-\left(t_{1 \pi}+t_{\mathrm{r}}\right)
\end{aligned}
$$


Fig. 3 displays the scrap level in condition one of the proposed system. It shows that the level of scrap accumulates to $d_{1} t$ at the time a breakdown happens, and after the breakdown repair is completed, it goes on to pile up to $d_{1} t_{1 \pi}$ in the end of uptime $t_{1 \pi}$.

$$
d_{1} t_{1 \pi}=x[(1-\pi) Q]=\left(x P_{1}\right) t_{1 \pi}
$$

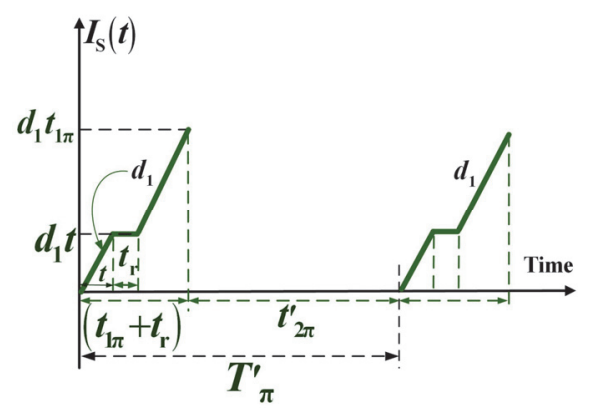

Fig. 3. The scrap level in condition 1 of the proposed system

Fig. 4 illustrates the finished stock level during $t^{\prime} 2 \pi$. Total holding stocks in $t^{\prime} 2 \pi$ can be calculated using Eq. (8) (Chiu et al., 2019c).

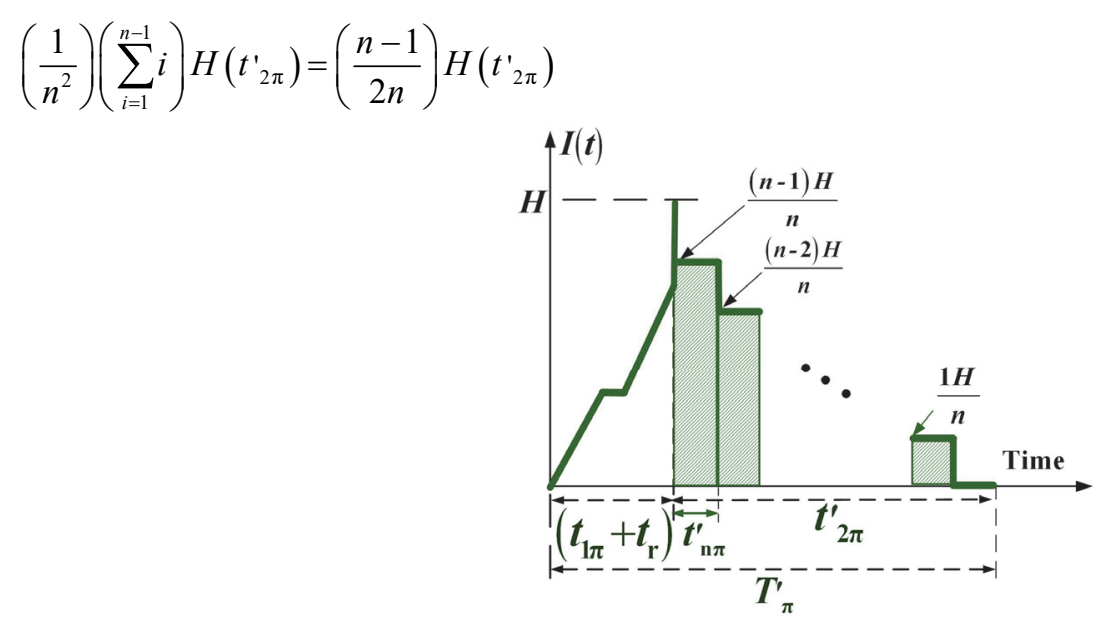

Fig. 4. The finished stock level during $t^{\prime} 2 \pi$ in condition 1 of the proposed system

The buyer's stock level is exhibited in Fig. 5, wherein $t^{\prime}{ }_{n \pi}, D$, and $I$ are shown in Eqs. (9) to (11) and total holding stocks in cycle time $T^{\prime}{ }_{\pi}$ can be computed by the use of Eq. (12) (Chiu et al., 2019c).

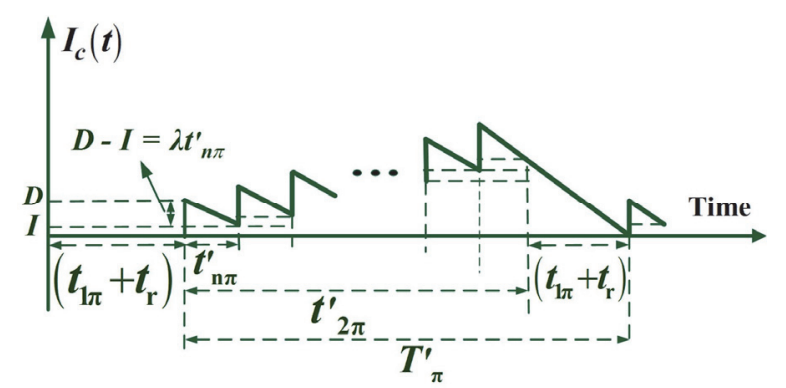

Fig. 5. The buyer stock level in the proposed system 


$$
\begin{aligned}
& t^{\prime}{ }_{n \pi}=\frac{t^{\prime}{ }_{2 \pi}}{n} \\
& D=\frac{H}{n} \\
& I=D-\left(\lambda t^{\prime}{ }_{n \pi}\right) \\
& n\left(t^{\prime}{ }_{n \pi}\right)\left(D-\frac{\lambda\left(t^{\prime}{ }_{n \pi}\right)}{2}\right)+\frac{n(n-1)}{2} I\left(t^{\prime}{ }_{n \pi}\right)+\frac{n I}{2}\left(t_{1 \pi}\right)=\frac{1}{2}\left[\frac{H t^{\prime}{ }_{2 \pi}}{n}+\left(H-\lambda t^{\prime}{ }_{2 \pi}\right) T^{\prime}{ }_{\pi}\right]
\end{aligned}
$$

Total cost per cycle in the condition 1 (i.e., a Poisson breakdown happening case), $T C\left(t_{1 \pi}\right)_{1}$ comprises both the variable and fixed outsourcing and in-house fabrication costs, breakdown repairing cost, safety stock related costs (refer to Fig. 2), fixed and variable transportation costs, disposal costs, and total holding costs (including buyer's stocks, in-house perfect and scrap items) during $T^{\prime} \pi$, as shown in Eq. (13).

$$
\begin{aligned}
& T C\left(t_{1 \pi}\right)_{1}=C_{\pi}(\pi Q)+K_{\pi}+C[(1-\pi) Q]+K+M+C_{1}\left(\lambda t_{r}\right)+h_{3}\left(\lambda t_{r}\right)\left(t_{1 \pi}+t_{r}\right) \\
& +n K_{1}+C_{T}\left[Q(1-x(1-\pi))+\lambda t_{r}\right]+C_{\mathrm{S}} x[(1-\pi) Q]+\frac{h_{2}}{2}\left[\frac{H t^{\prime}{ }_{2 \pi}}{n}+\left(H-\lambda t^{\prime}{ }_{2 \pi}\right) T^{\prime}{ }_{\pi}\right] \\
& +h\left[\frac{H_{1}+d_{1} t_{1 \pi}}{2}\left(t_{1 \pi}\right)+\left(H_{0} t_{r}\right)+\left(d_{1} t\right) t_{r}+\left(\frac{n-1}{2 n}\right) H t_{2 \pi}^{\prime}\right]
\end{aligned}
$$

Substitute Eq. (1) to Eq. (12) in Eq. (13), and employ the expected value to cope with the randomness of $x$, the following $E\left[T C\left(t_{1 \pi}\right)_{1}\right]$ can be derived:

$$
\begin{aligned}
E[ & \left.T C\left(t_{1 \pi}\right)_{1}\right]=C_{\pi}\left[\frac{\pi t_{1 \pi} P_{1}}{(1-\pi)}\right]+K_{\pi}+C\left(t_{1 \pi} P_{1}\right)+K+M+n K_{1}+C_{T}\left[\frac{t_{1 \pi} P_{1}}{(1-\pi)} y_{0}+\lambda g\right] \\
& +C_{1} \lambda g+h_{3}\left(\lambda g t_{1 \pi}+\lambda g^{2}\right)+C_{S} E[x] t_{1 \pi} P_{1}+h\left(P_{1} t g\right)+\frac{t_{1 \pi}^{2} P_{1}^{2}\left(h_{2}-h\right)}{2 n \lambda(1-\pi)} y_{0}\left(y_{1}-y_{2}\right) \\
& +h\left[\frac{g t_{1 \pi} P_{1}}{2}\left(y_{1}-y_{2}\right)\right]+\frac{h t_{1 \pi}^{2} P_{1}^{2}}{2 \lambda(1-\pi)}\left\{\frac{y_{0}^{2}}{(1-\pi)}+\frac{\lambda[E[x](1-\pi)-\pi]}{P_{1}}\right\}+h_{2}\left(\frac{\lambda g^{2}}{2}\right) \\
& +h_{2}\left[\frac{g t_{1 \pi} P_{1}}{2}\left(y_{1}+y_{2}\right)\right]+\frac{h_{2} t_{1 \pi}^{2} P_{1} y_{0}}{2(1-\pi)}+\left(h_{2}-h\right)\left[\frac{g t_{1 \pi} P_{1}}{2 n}\left(y_{1}-y_{2}\right)\right]
\end{aligned}
$$

where

$$
y_{0}=[1-E[x](1-\pi)] ; y_{1}=\left[\frac{1}{(1-\pi)}-E[x]\right] ; y_{2}=\left(\frac{\lambda}{P_{1}}\right) .
$$

The following $E\left[T^{\prime} \pi\right]$ can be gained by employing $E[x]$ to manage random scrap rate:

$$
E\left[T^{\prime}{ }_{\pi}\right]=\frac{Q[1-E[x](1-\pi)]+\lambda t_{r}}{\lambda}=\frac{t_{1 \pi} P_{1} y_{1}+\lambda t_{r}}{\lambda}
$$

\subsubsection{Condition 2: No breakdown happens during $t_{1 \pi}$}

In condition two, $t \geq t_{1 \pi}$. Fig. 6 displays the finished stock level in condition two of the proposed system. Fig. 6 explicitly indicates that the finished stock arrives at $H_{1}$ in the end of uptime, prior to the beginning of distribution time $t_{2 \pi}$, the outsourced products are received, which bring the finished stock level to $H$. Hence, we directly observe the following formulas:

$$
H_{1}=\left(P_{1}-d_{1}\right) t_{1 \pi}
$$




$$
\begin{aligned}
& H=H_{1}+\pi Q \\
& T_{\pi}=t_{1 \pi}+t_{2 \pi} \\
& t_{1 \pi}=\frac{Q(1-\pi)}{P_{1}}
\end{aligned}
$$

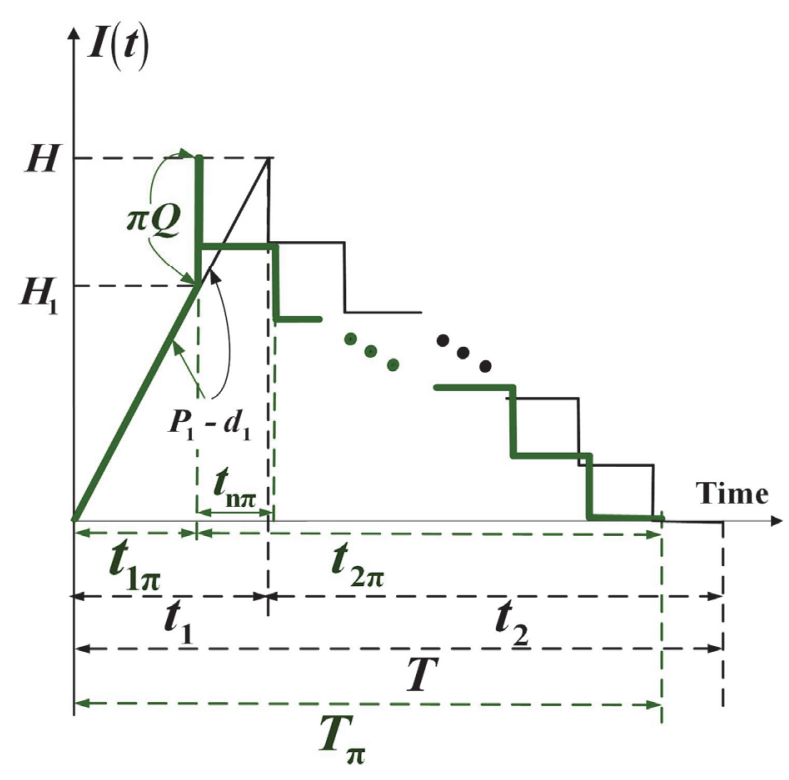

Fig. 6. The finished stock level in condition two of the proposed system (in green) as compared to that of the proposed system without outsourcing plan (in black)

Fig. 7 shows the safety stock level in condition two of the proposed system. Since there is no breakdown happening, it remains the same throughout $T_{\pi}$.

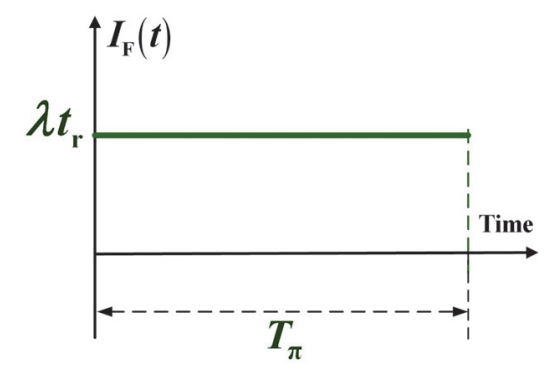

Fig. 7. The safety stock level in condition two of the proposed system

Similar to that in condition one (see Fig. 3 to Fig. 5), the scrap, finished stock, and buyer stock levels in condition two of the proposed system is as follows (Chiu et al., 2019c):

$$
\begin{aligned}
& d_{1} t_{1 \pi}=x[(1-\pi) Q]=\left(x P_{1}\right) t_{1 \pi} \\
& \left(\frac{1}{n^{2}}\right)\left(\sum_{i=1}^{n-1} i\right) H\left(t_{2 \pi}\right)=\left(\frac{n-1}{2 n}\right) H\left(t_{2 \pi}\right) \\
& \frac{1}{2}\left[\frac{H t_{2 \pi}}{n}+\left(H-\lambda t_{2 \pi}\right) T_{\pi}\right]
\end{aligned}
$$


Therefore, in condition two, the following $T C\left(t_{1 \pi}\right)_{2}$ comprises both the variable and fixed outsourcing and in-house fabrication costs, holding cost for safety stock, variable and fixed transportation costs, disposal costs, and total holding costs (including buyer's stocks, in-house perfect and scrap items) during $T_{\pi}$ :

$$
\begin{aligned}
& T C\left(t_{1 \pi}\right)_{2}=C_{\pi}(\pi Q)+K_{\pi}+C[(1-\pi) Q]+K+h_{3}\left(\lambda t_{r}\right) T_{\pi}+C_{T} Q[1-x(1-\pi)] \\
& +n K_{1}+C_{\mathrm{S}} x[(1-\pi) Q]+\frac{h_{2}}{2}\left[\frac{H t_{2 \pi}}{n}+\left(H-\lambda t_{2 \pi}\right) T_{\pi}\right]+h\left[\frac{H_{1}+d_{1} t_{1 \pi}}{2}\left(t_{1 \pi}\right)+\left(\frac{n-1}{2 n}\right) H t_{2 \pi}\right]
\end{aligned}
$$

Substitute Eq. (16) to Eq. (22) in Eq. (23), and employ the expected value to cope with the randomness of $x$, the following expected total system cost per cycle $E\left[T C\left(t_{1 \pi}\right)_{2}\right]$ can be obtained:

$$
\begin{aligned}
& E\left[T C\left(t_{1 \pi}\right)_{2}\right]=C_{\pi}\left[\frac{\pi t_{1 \pi} P_{1}}{(1-\pi)}\right]+K_{\pi}+C\left(t_{1 \pi} P_{1}\right)+K+h_{3}\left(g t_{1 \pi} P_{1} y_{1}\right)+C_{S} E[x] t_{1 \pi} P_{1}+C_{T} t_{1 \pi} P_{1} y_{1} \\
& +n K_{1}+\frac{t_{1 \pi}^{2} P_{1}^{2}\left(h_{2}-h\right) y_{1}}{2 n \lambda}\left(y_{1}-y_{2}\right)+\frac{h t_{1 \pi}^{2} P_{1}^{2}}{2 \lambda(1-\pi)}\left\{\frac{y_{0}{ }^{2}}{(1-\pi)}+\frac{\lambda[E[x](1-\pi)-\pi]}{P_{1}}\right\}+\frac{h_{2} t_{1 \pi}^{2} P_{1} y_{0}}{2(1-\pi)}
\end{aligned}
$$

The following $E\left[T_{\pi}\right]$ can be gained by employing $E[x]$ to manage random scrap rate:

$$
E\left[T_{\pi}\right]=\frac{Q[1-E[x](1-\pi)]}{\lambda}=\frac{t_{1 \pi} P_{1} y_{1}}{\lambda}
$$

\section{Solution procedure}

Due to the assumption of Poisson breakdown rate $\beta$ per year, the time to breakdown obeys the Exponential distribution with $f(t)=\beta e^{-\beta t}$ and $\mathrm{F}(t)=\left(1-e^{-\beta t}\right)$. Also, the cycle time is not constant due to the random scrap rate. The renewal reward theorem is applied here to deal with the variable cycle time. So, the following $E\left[T C U\left(t_{1 \pi}\right)\right]$ can be calculated:

$$
E\left[T C U\left(t_{1 \pi}\right)\right]=\frac{\left\{\int_{0}^{t_{1 \pi}} E\left[T C\left(t_{1 \pi}\right)_{1}\right] \cdot f(t) d t+\int_{t_{1 \pi}}^{\infty} E\left[T C\left(t_{1 \pi}\right)_{2}\right] \cdot f(t) d t\right\}}{E\left[\boldsymbol{T}_{\pi}\right]},
$$

where

$$
E\left[\boldsymbol{T}_{\pi}\right]=\int_{0}^{t_{1 \pi}} E\left[T^{\prime}{ }_{\pi}\right] \cdot f(t) d t+\int_{t_{1 \pi}}^{\infty} E\left[T_{\pi}\right] \cdot f(t) d t
$$

Substitute formulas (14), (24), and (27) in formula (26), along with some efforts in derivations, $E\left[T C U\left(t_{1 \pi}\right)\right]$ is derived as follows (please see Appendix A for details):

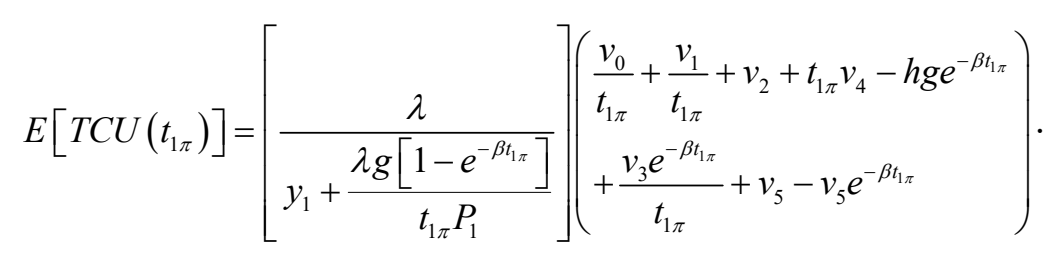

The first- and second-derivatives of $E\left[T C U\left(t_{1} \pi\right)\right]$ are shown in Eqs. (B-1) and (B-2) in Appendix B. Sinc e the first term on the right-hand side (RHS) of Eq. (B-2) is positive, it follows that the $E\left[T C U\left(t_{1 \pi}\right)\right]$ is c onvex if the second term on the RHS of Eq. (B-2) is also positive. That means if $\delta\left(t_{1 \pi}\right)>t_{1 \pi}>0$ holds (s ee Eq. (B-3) for details). If Eq. (B-3) holds, $t_{1 \pi} *$ can be solved by letting the first-derivative of $E\left[T C U\left(t_{1}\right.\right.$ $\pi)]=0$. Since the first term on the RHS of Eq. (B-1) is positive, we obtain the following: 


$$
\left\{\begin{array}{l}
{\left[\left(h g+v_{5}\right) P_{1}\left(y_{1} P_{1} \beta e^{-\beta t_{1 \pi}}\right)+v_{4} P_{1}\left(y_{1} P_{1}-\lambda g \beta e^{-\beta t_{1 \pi}}\right)\right] t_{1 \pi}{ }^{2}} \\
+\left[v_{3} P_{1}\left(-y_{1} P_{1} \beta e^{-\beta t_{1 \pi}}\right)+v_{4} P_{1}\left(2 \lambda g-2 \lambda g e^{-\beta t_{1 \pi}}\right)+\left(h g-v_{2}\right) P_{1} \lambda g\left(\beta e^{-\beta t_{1 \pi}}\right)\right] t_{1 \pi} \\
-\left(v_{0}+v_{1}\right) P_{1}\left(y_{1} P_{1}+\lambda g \beta e^{-\beta t_{1 \pi}}\right)+v_{3} P_{1}\left(-\lambda g \beta e^{-\beta t_{1 \pi}}-y_{1} P_{1} e^{-\beta t_{1 \pi}}\right) \\
-\left(h g+v_{5}\right) P_{1} \lambda g\left(-e^{-2 \beta t_{1 \pi}}+e^{-\beta t_{1 \pi}}\right)-\left(v_{2}+v_{5}\right) P_{1} \lambda g\left(e^{-\beta t_{1 \pi}}-1\right)
\end{array}\right\}=0
$$

Let $\gamma_{0}, \gamma_{1}$, and $\gamma_{2}$ represent the following:

$$
\begin{aligned}
\gamma_{0}= & {\left[\left(h g+v_{5}\right) P_{1}\left(y_{1} P_{1} \beta e^{-\beta t_{1 \pi}}\right)+v_{4} P_{1}\left(y_{1} P_{1}-\lambda g \beta e^{-\beta t_{1 \pi}}\right)\right] } \\
\gamma_{1}= & {\left[v_{3} P_{1}\left(-y_{1} P_{1} \beta e^{-\beta t_{1 \pi}}\right)+v_{4} P_{1}\left(2 \lambda g-2 \lambda g e^{-\beta t_{1 \pi}}\right)+\left(h g-v_{2}\right) P_{1} \lambda g\left(\beta e^{-\beta t_{1 \pi}}\right)\right] } \\
\gamma_{2}= & -\left(v_{0}+v_{1}\right) P_{1}\left(y_{1} P_{1}+\lambda g \beta e^{-\beta t_{1 \pi}}\right)+v_{3} P_{1}\left(-\lambda g \beta e^{-\beta t_{1 \pi}}-y_{1} P_{1} e^{-\beta t_{1 \pi}}\right) \\
& -\left(h g+v_{5}\right) P_{1} \lambda g\left(-e^{-2 \beta t_{1 \pi}}+e^{-\beta t_{1, \pi}}\right)-\left(v_{2}+v_{5}\right) P_{1} \lambda g\left(e^{-\beta t_{1 \pi}}-1\right)
\end{aligned}
$$

Then, we can rearrange Eq. (33) as follows:

$$
\gamma_{0}\left(t_{1 \pi}\right)^{2}+\gamma_{1}\left(t_{1 \pi}\right)+\gamma_{2}=0
$$

Apply the square roots solution, $t_{\pi}^{*}$ can be found as follows:

$$
t_{1 \pi}^{*}=\frac{-\gamma_{1} \pm \sqrt{\gamma_{1}^{2}-4 \gamma_{0} \gamma_{2}}}{2 \gamma_{0}}
$$

\subsection{Recursive algorithm for finding $t_{1 \pi} *$}

As $F\left(t_{1 \pi}\right)=\left(1-e^{-\beta t 1 \pi}\right)$ is over the interval of [0.1], so does its complement $e^{-\beta t 1 \pi}$. So, Eq. (31) can be rearranged as follows:

$$
e^{-\beta t_{1 \pi}}=\frac{-v_{4} t_{1 \pi} P_{1}\left(y_{1} P_{1}-2 \lambda g\right)+\left(v_{0}+v_{1}\right) P_{1}^{2} y_{1}-\left(v_{2}+v_{5}\right) P_{1} \lambda g}{\left\{\begin{array}{l}
\left(h g+v_{5}\right) P_{1}^{2} y_{1} \beta t_{1 \pi}{ }^{2}+\left[-v_{3} P_{1}^{2} y_{1} \beta-v_{4} t_{1 \pi} P_{1} \lambda g \beta+\left(h g-v_{2}\right) P_{1} \lambda g \beta\right] t_{1 \pi} \\
-\left[\left(v_{2}+v_{5}\right) P_{1} \lambda g\right]-\left[\left(h g+v_{5}\right) P_{1} \lambda g\left(1-e^{-\beta t_{1 \pi}}\right)\right]-2 v_{4} t_{1 \pi} P_{1} \lambda g \\
-\left[\left(v_{0}+v_{1}\right) P_{1}(\lambda g \beta)\right]+\left[v_{3} P_{1}\left(-\lambda g \beta-y_{1} P_{1}\right)\right]
\end{array}\right\}}
$$

The following recursive algorithm is proposed to find optimal $t_{1} \pi^{*}$ :

(i) Let $e^{-\beta t 1 \pi}=0$ and $e^{-\beta t 1 \pi}=1$, apply Eq. (31) to obtain the bounds for $t_{1 \pi} *$ first (i.e., $t_{1 \pi \mathrm{U}}$ and $t_{1 \pi \mathrm{L}}$ ).

(ii) Use the current values of $t_{1 \pi \mathrm{U}}$ and $t_{1 \pi \mathrm{L}}$ to calculate the update values of $e^{-\beta t 1 \pi \mathrm{U}}$ and $e^{-\beta t 1 \pi \mathrm{L}}$.

(iii) Re-apply Eq. (31) using the current $e^{-\beta t 1 \pi \mathrm{U}}$ and $e^{-\beta t 1 \pi \mathrm{L}}$ to gain the update values of $t_{1 \pi \mathrm{U}}$ and $t_{1 \pi \mathrm{L}}$.

(iv) Test to see if $t_{1 \pi \mathrm{U}}=t_{1 \pi \mathrm{L}}$ ? If yes, then $t_{1 \pi} *$ is derived, that is $t_{1 \pi} *=t_{1 \pi \mathrm{L}}=t_{1 \pi \mathrm{U}}$; otherwise, goes to step (ii).

\section{Numerical illustration}

A numerical example is offered to demonstrate how our proposed solution procedure works and the assumption of relevant parameters is exhibited as follows (see Table 1):

Table 1

Assumption of relevant parameters

\begin{tabular}{ccccccccccc}
\hline$C_{\mathrm{T}}$ & $K_{1}$ & $\pi$ & $K$ & $\lambda$ & $\beta$ & $C$ & $C_{1}$ & $\beta_{2}$ & $P_{1}$ & $C_{\mathrm{S}}$ \\
\hline 0.01 & 90 & 0.4 & 200 & 4000 & 1 & 2.0 & 2.0 & 0.4 & 10000 & 0.1 \\
$h_{2}$ & $n$ & $C_{\pi}$ & $K_{\pi}$ & $\mathrm{M}$ & $x$ & $h$ & $h_{3}$ & $\beta_{1}$ & $g$ & \\
1.6 & 3 & 2.8 & 60 & 2500 & $20 \%$ & 0.4 & 0.4 & -0.70 & 0.018 & \\
\hline
\end{tabular}


The solution procedure starts with its prerequisite, that is to make sure $E\left[T C U\left(t_{1 \pi}\right)\right]$ is convex. For $e^{-\beta t 1 \pi}$ falls within the range $[0,1]$, we start with setting $e^{-\beta t 1 \pi}=0$ and $e^{-\beta t 1 \pi}=1$, and apply Eq. (31) to gain the initial $t_{1 \pi \mathrm{L}}=0.0940$ and $t_{1 \pi \mathrm{U}}=0.3012$. Then, we use the obtained $t_{1 \pi \mathrm{L}}$ and $t_{1 \pi \mathrm{U}}$ to compute $e^{-\beta t 1 \pi \mathrm{L}}$ and $e^{-}$ $\beta t 1 \pi \mathrm{U}$. Lastly, we apply Eq. (B-3) with the present values of $e^{-\beta t 1 \pi \mathrm{L}}, e^{-\beta t 1 \pi \mathrm{U}}, t_{1 \pi \mathrm{L}}$, and $t_{1 \pi \mathrm{U}}$ to confirm that $\delta\left(t_{1 \pi \mathrm{L}}\right)=0.3139>t_{1 \pi \mathrm{L}}=0.0940>0$ and $\delta\left(t_{1 \pi \mathrm{U}}\right)=0.5480>t_{1 \pi \mathrm{U}}=0.3012>0$. Thus, for $\beta=1$ we confirm the convexity of $E\left[T C U\left(t_{1 \pi}\right)\right]$, so the optimal $t_{1 \pi} *$ does exist. In addition, a broader range of $\beta$ values are used for testing convexity of $E\left[T C U\left(t_{1 \pi}\right)\right]$ to show the applicability of our proposed model, and the outcomes are displayed in Table C-1 (see Appendix C). For locating $t_{1}{ }^{*}$, we apply the proposed recursive algorithm which was mentioned in previous subsection. Table 2 illustrates the detailed iterative results of the $t_{1 \pi} *$ searching algorithm. It indicates that in our example (i.e., $\beta=1$ ) the optimal uptime $t_{1 \pi} *=$ 0.1283 and $E\left[T C U\left(t_{1 \pi}^{*}\right)\right]=\$ 12,664.59$.

\section{Table 2}

Detailed iterative results of the searching algorithm for $t_{1 \pi} *$

\begin{tabular}{cccccccc}
\hline $\begin{array}{c}\text { Iteration } \\
\text { number }\end{array}$ & $t_{1 \pi \mathrm{U}}$ & $e^{-\beta t 1 \pi \mathrm{U}}$ & $t_{1 \pi \mathrm{L}}$ & $e^{-\beta t 1 \pi \mathrm{L}}$ & $t_{1 \pi \mathrm{U}-t_{1 \pi \mathrm{L}}}$ & $E\left[T C U\left(t_{1 \pi \mathrm{U})}\right]\right.$ & $E\left[T C U\left(t_{1 \pi \mathrm{L})}\right]\right.$ \\
\hline- & - & 0 & - & 1 & - & - & - \\
1 & $\mathbf{0 . 3 0 1 2}$ & 0.7399 & $\mathbf{0 . 0 9 4 0}$ & 0.9103 & 0.2072 & $\$ 13,476.19$ & $\$ 12,766.25$ \\
2 & 0.1624 & 0.8501 & 0.1201 & 0.8868 & 0.0423 & $\$ 12,722.17$ & $\$ 12,668.19$ \\
3 & 0.1359 & 0.8729 & 0.1264 & 0.8812 & 0.0095 & $\$ 12,667.08$ & $\$ 12,663.83$ \\
4 & 0.1301 & 0.8780 & 0.1279 & 0.8799 & 0.0022 & $\$ 12,663.78$ & $\$ 12,663.60$ \\
5 & 0.1287 & 0.8792 & 0.1282 & 0.8796 & 0.0005 & $\$ 12,663.60$ & $\$ 12,663.59$ \\
6 & 0.1284 & 0.8795 & 0.1283 & 0.8796 & 0.0001 & $\$ 12,663.59$ & $\$ 12,663.59$ \\
7 & $\mathbf{0 . 1 2 8 3}$ & 0.8796 & $\mathbf{0 . 1 2 8 3}$ & 0.8796 & $\mathbf{0 . 0 0 0 0}$ & $\mathbf{\$ 1 2 , 6 6 3 . 5 9}$ & $\mathbf{\$ 1 2 , 6 6 3 . 5 9}$ \\
\hline
\end{tabular}

Fig. 8 illustrates the initial bounds for $t_{1 \pi}$, convexity, and the effect of deviations in $t_{1 \pi}$ on $E\left[T C U\left(t_{1 \pi}\right)\right]$.

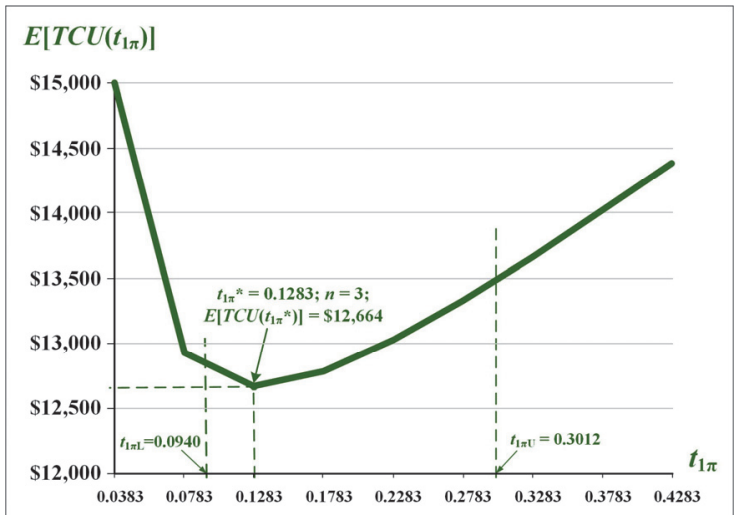

Fig. 8. Initial bounds for $t_{1 \pi}$, the convexity, and the effect of deviations in $t_{1 \pi}$ on $E\left[T C U\left(t_{1 \pi}\right)\right]$

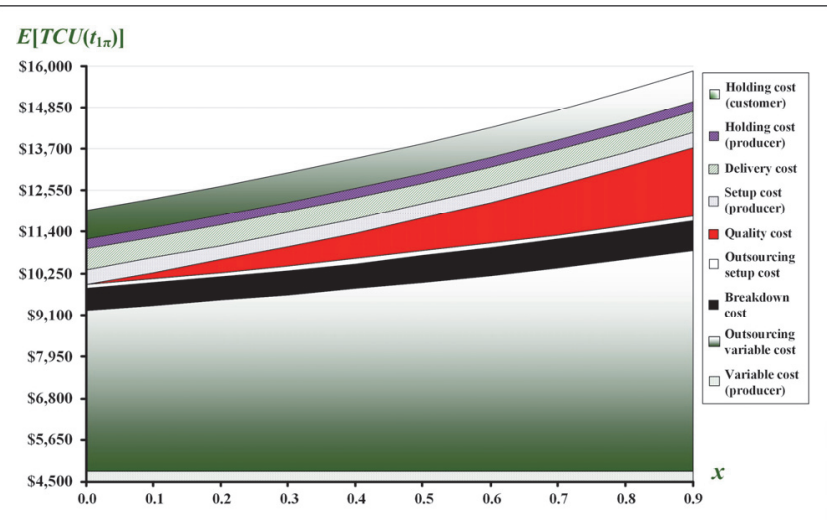

Fig. 9. Variations in random scrap rate $x$ effect on different system cost contributors

The variations in scrap rate $x$ effect on different system cost contributors are explored and depicted in Fig. 9. It indicates that product quality relevant cost (including disposal cost and the expense for fabricating extra items to make up the scrap) upsurges drastically, as $x$ increases.

The impact of differences in mean-time-to-breakdown $1 / \beta$ along with various values of $x$ on $E\left[T C U\left(t_{1} \pi^{*}\right)\right]$ is investigated as shown in Figure 10 . It specifies that for $1 / \beta=1, x=0.2$, and $n=3$, we have $E\left[T C U\left(t_{1 \pi^{*}}\right)\right]=\$ 12,664$; it also reveals that $E\left[T C U\left(t_{1 \pi^{*}}\right)\right]$ starts to drastically decline, when $1 / \beta$ rises to and beyond 0.14 . Moreover, once $1 / \beta$ grows into very large (e.g., $1 / \beta$ approaches 100), $E\left[T C U\left(t_{1} \pi^{*}\right)\right]=\$ 12,067$ (i.e., the same result as that of a problem without considering breakdown happening). 


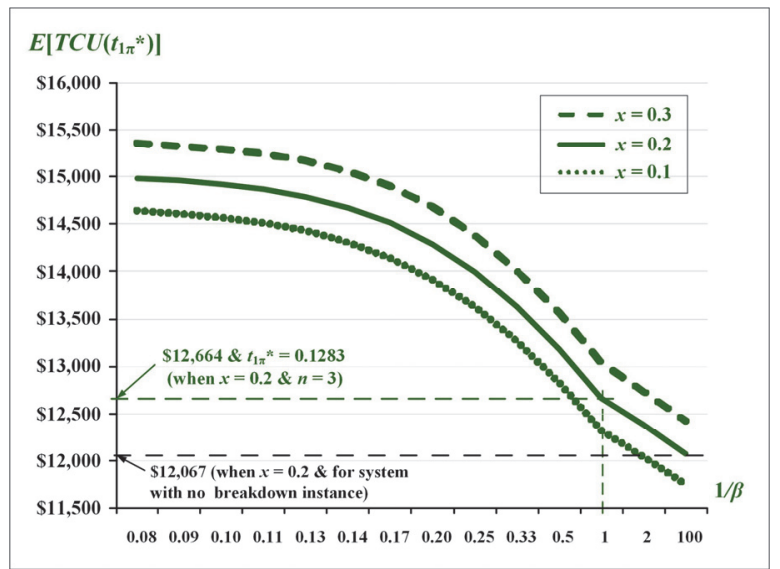

Fig. 10. The impact of differences in $1 / \beta$ along with $x$ on $E\left[T C U\left(t_{\left.\left.1 \pi^{*}\right)\right]}\right.\right.$

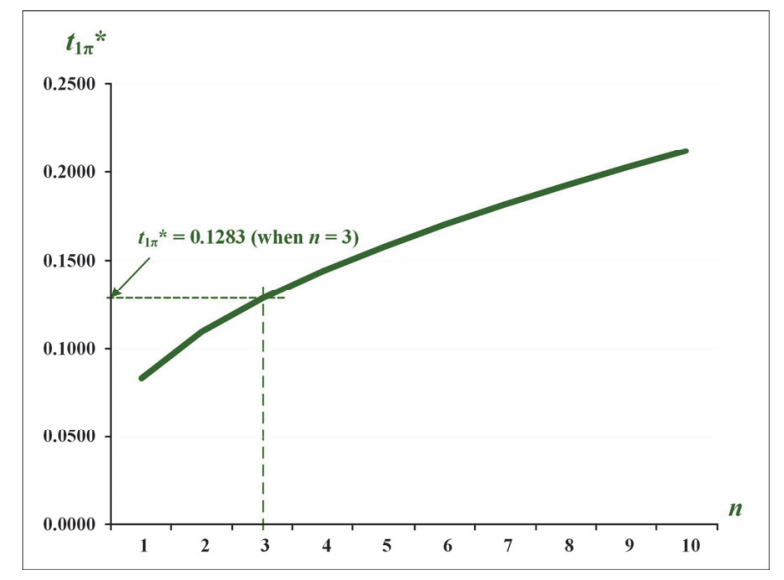

Fig. 11. Effect of changes in $n$ on $t_{1 \pi^{*}}$

The effect of changes in number of shipments in a cycle $n$ on optimal uptime $t_{1 \pi} *$ is exhibited in Fig. 11. It specifies that in our example $n=3, t_{1 \pi^{*}}=0.1283$; and $t_{1 \pi^{*}}$ increases considerably, as $n$ rises. The influence of variations in number of shipments in a cycle $n$ on the delivery relevant costs is illustrated in Fig. 12. It indicates that as $n$ increases, the fixed delivery cost upsurges severely and in-house holding cost goes up accordingly (the latter is due to a slow movement of goods from producer to customer as $n$ increases); on the contrary, customer's holding cost varies slightly, except for $n=1$.

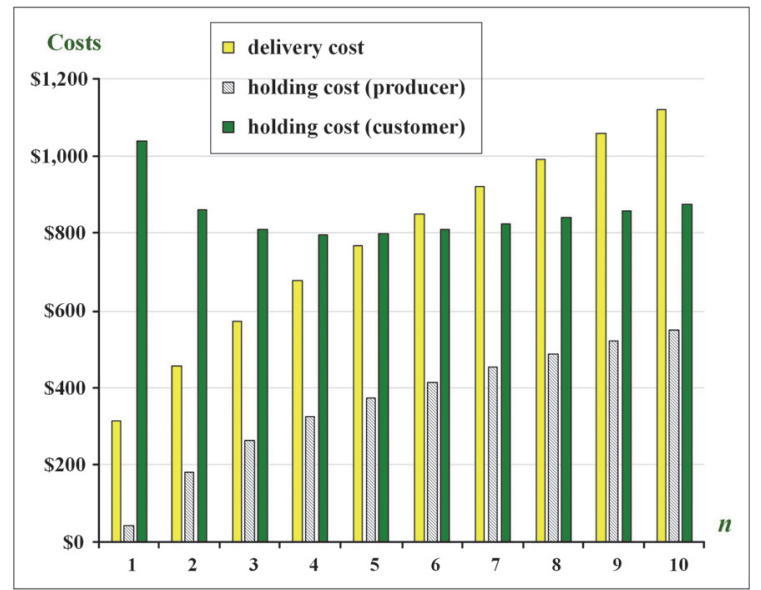

Fig. 12. Influence of variations in $n$ on the delivery relevant costs

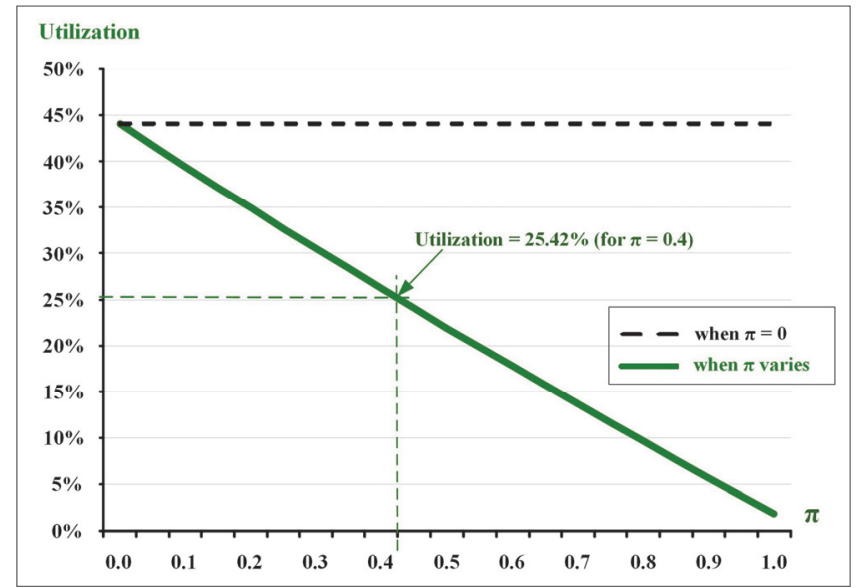

Fig. 13. Impact of differences in $\pi$ on utilization

The impact of differences in outsourcing portion $\pi$ on utilization is demonstrated in Fig. 13. It specifies that for $\pi=0.4$ (our assumption in the example), utilization drops from $44.12 \%$ to $25.42 \%$; and utilization declines significantly as $\pi$ increases.

The effect of variations in outsourcing portion $\pi$ on diverse cost contributors of $E\left[T C U\left(T_{1 \pi} *\right)\right]$ are investigated and exposed in Fig. 14. It shows that as $\pi$ increases, in-house variable cost decreases severely, the quality and breakdown costs also declines noticeably; quite the reverse, variable outsourcing cost upsurges radically. Fig. 15 illustrates the combined influences of changes in extra fraction of unit outsourcing cost $\beta_{2}$ and scrap rate $x$ on $E\left[T C U\left(t_{1} \pi^{*}\right)\right]$. It reveals that $x$ has more influence on the expected system cost than $\beta_{2}$; for $E\left[T C U\left(t_{1} \pi^{*}\right)\right]$ upsurges radically as $x$ goes up; and it increases mildly as $\beta_{2}$ rises. The joint effect of variations in outsourcing portion $\pi$ and random scrap rate $x$ on the optimal decision variable $t_{1 \pi} *$ is analyzed and demonstrated in Figure 16. It exposes that $\pi$ has more impact on the optimal 
$t_{1 \pi} *$ than $x$. The decision variable $t_{1 \pi} *$ declines radically as $\pi$ increases; and conversely when $x$ increases, $t_{1 \pi} *$ noticeable rises only when $\pi$ is small (e.g., less than 0.4 ), otherwise, $t_{1 \pi} *$ increases insignificantly, as $x$ rises.

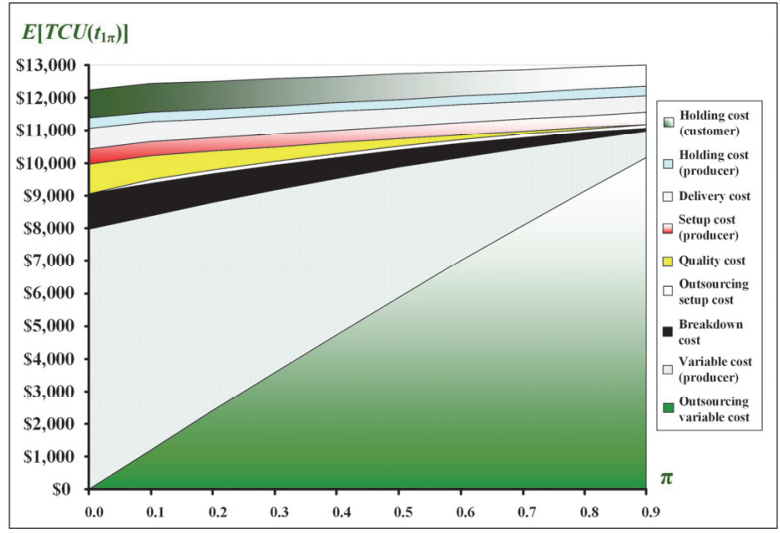

Fig. 14. Effect of variations in $\pi$ on diverse cost contributors of $E\left[T C U\left(t_{1 \pi}{ }^{*}\right)\right]$

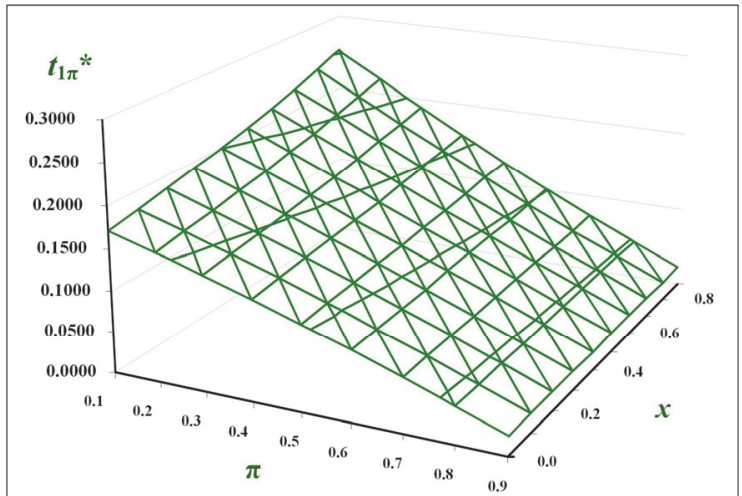

Fig. 16. Joint effect of variations in $\pi$ and $x$ on $t_{1} \pi^{*}$

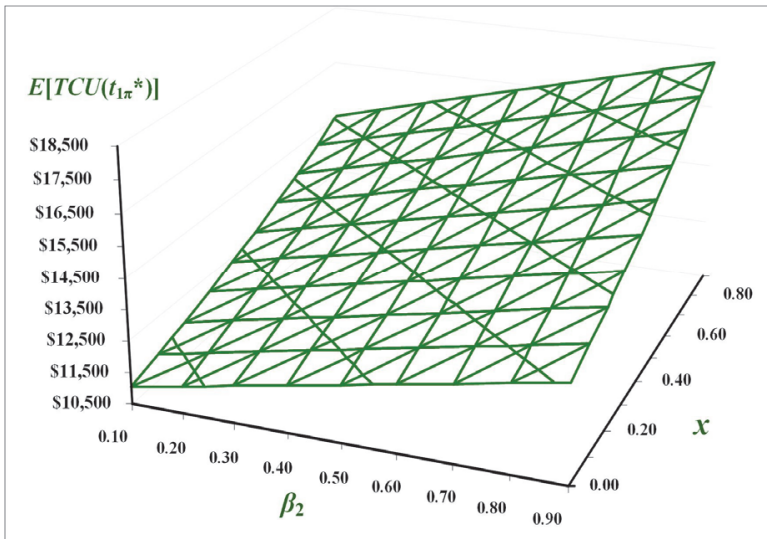

Fig. 15. Combined influences of changes in $\beta_{2}$ and $x$ on $E\left[T C U\left(t_{1 \pi}{ }^{*}\right)\right]$

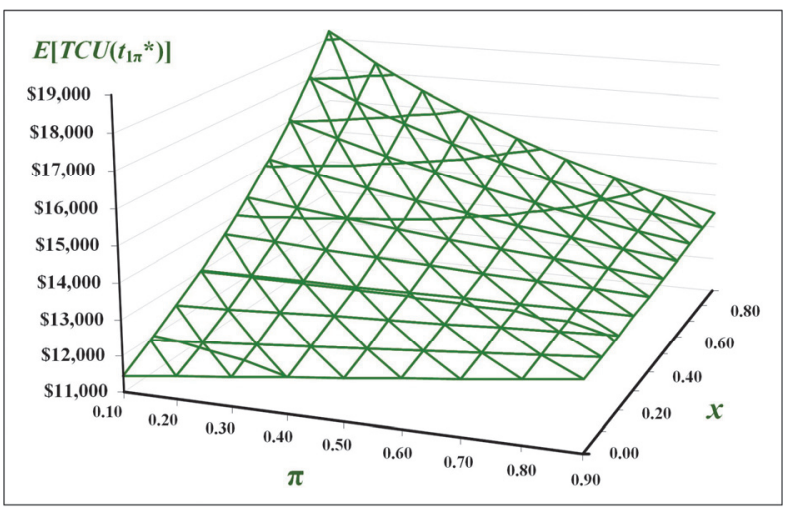

Fig. 17. Combined impact of differences in $x$ and $\pi$ on the optimal $E\left[T C U\left(t_{1} \pi^{*}\right)\right]$

Fig. 17 exhibits the combined impact of differences in scrap rate $x$ and outsourcing portion $\pi$ on optimal system cost $E\left[T C U\left(t_{1 \pi^{*}}\right)\right]$. It reveals that $x$ has more influence on $E\left[T C U\left(t_{1} \pi^{*}\right)\right]$ than $\pi$, especially when $\pi$ is less than 0.6 (i.e., $E\left[T C U\left(t_{1} \pi^{*}\right)\right]$ upsurges drastically as $x$ goes up); as $\pi>0.6, E\left[T C U\left(t_{1} \pi^{*}\right)\right]$ increases mildly as $x$ rises. On the other hand, when $x$ is small, as $\pi$ rises, $E\left[T C U\left(t_{1} \pi^{*}\right)\right]$ surges accordingly; and in contrast, when $x$ is high, $E\left[T C U\left(t_{1 \pi} *\right)\right]$ declines significantly, as $\pi$ increases.

\section{Conclusions}

The present work has explored a vendor-buyer coordinated system featuring an unreliable machine, scrap, outsourcing, and multi-shipment policy. A decision support type of model was built to clearly portray the characteristics of the problem to help vendor gain the competitive advantage by ensuring the timeliness and quality-product deliveries to buyer given unreliable machine and limited capacity. By the use of mathematical analyses, derivations, optimization processes, and a specific recursive algorithm, we are able to obtain the optimal expected system cost and fabrication uptime decision to the problem. The applicability of research result is demonstrated through numerical illustrations. Diverse hidden information of the problem that can facilitate managerial decision making is now revealed, it includes: (i) the influence of deviations in $t_{1 \pi}$ on $E\left[T C U\left(t_{1 \pi}\right)\right]$ (Fig. 8); (ii) the effect of differences in $x$ on various cost contributors of the system (Fig. 9); (iii) the impact of changes in $1 / \beta$ along with $x$ on $E\left[T C U\left(t_{11} \pi^{*}\right)\right]$ (Fig. 10); (iv) the influence of variations in $n$ on $t_{1} \pi^{*}$ and various delivery relevant costs (Figs. 11-12); 
(v) the impact of differences in $\pi$ on utilization (Fig. 13); (vi) the effect of changes in $\pi$ on various cost contributors of $E\left[T C U\left(t_{1} \pi^{*}\right)\right]$ (Fig. 14); and (vii) the joint impacts of variations in key system parameters on the optimal decision of the problem (Figs. 15-17). For future study, incorporating a stochastic demand in the same context of the problem will be an interesting direction.

\section{Acknowledgment}

The authors appreciate the Ministry of Science and Technology (Taiwan) for sponsoring this research (Grant\#: MOST 107-2221-E-324-015).

\section{References}

Al-Odeh, M., \& Altarazi, S. (2019). Supply chain management information systems survey for Jordanian companies. International Journal of Mathematical, Engineering and Management Sciences, 4(3), 567-579.

Alam, M., \& Sarma, V.V.S. (1974). Optimum maintenance policy for an equipment subject to deterioration and random failure. IEEE Transactions on Systems, Man and Cybernetics, SMC-4(2), 172-175.

Alvarez-Vergas, R., Dallery, Y., \& David, R. (1994). A study of the continuous flow-model of production lines with unreliable machines and finite buffers. Journal of Manufacturing Systems, 13(3), 221-234.

Bryce, D.J., \& Useem, M. (1998). The impact of corporate outsourcing on company value. European Management Journal, 16(6), 635-643.

Chakravarthy, S. (1983). Reliability analysis of a parallel system with exponential life times and phase type repairs. OR Spektrum, 5(1), 25-32.

Chiu, Y-S.P., Liu, C-J., \& Hwang, M-H. (2017). Optimal batch size considering partial outsourcing plan and rework. Jordan Journal of Mechanical and Industrial Engineering, 11(3), 195-200.

Chiu, Y-S.P., Chen, Y-R., Chiu, V., \& Chiu, S.W. (2019a). Joint effects of stochastic machine failure, backorder of permissible shortage, rework, and scrap on stock replenishing decision. International Journal of Industrial Engineering Computations, 10(2), 263-280.

Chiu, Y-S.P., Chiu, V., Lin, H-D., \& Chang, H-H. (2019b). Meeting multiproduct demand with a hybrid inventory replenishment system featuring quality reassurance. Operations Research Perspectives, 6 , Art. No. 100112, 1-8.

Chiu, S.W., Wu, C-S., \& Tseng, C-T. (2019c). Incorporating an expedited rate, rework, and a multishipment policy into a multi-item stock refilling system. Operations Research Perspectives, 6, Art. No. 100115, 1-12.

Çınar, E., \& Güllü, R. (2012). An inventory model with capacity flexibility in the existence of advance capacity information. Decision Support Systems, 53, 320-330.

Díaz-Mateus, Y., Forero, B., López-Ospina, H., \& Zambrano-Rey, G. (2018). Pricing and lot sizing optimization in a two-echelon supply chain with a constrained logit demand function. International Journal of Industrial Engineering Computations, 9(2), 205-220.

Golmakani, H.R., Moakedi, H. (2012). Periodic inspection optimization model for a two-component repairable system with failure interaction. Computers and Industrial Engineering, 63(3), 540-545.

Hill, R.M. (1996). Optimizing a production system with a fixed delivery schedule. Journal of the Operational Research Society, 47, 954-960.

Kamien, M.I., \& Li, L. (1990). Subcontracting, Coordination, Flexibility, and Production Smoothing in Aggregate Planning. Management Science, 36(11), 1352-1363.

Köksal, G., Taşeli, A., Dolgun, L.E., \& Batmaz, I. (2013). The effect of inspection error on quality and producer losses: The case of nominal-the-best type quality characteristic and rework European Journal of Industrial Engineering, 7(4), 497-528.

Kuhn, H., \& Liske, T. (2011). Simultaneous supply and production planning. International Journal of Production Research, 49(13), 3795-3813.

Lee, I.S., \& Sung, C.S. (2008). Single machine scheduling with outsourcing allowed. International Journal of Production Economics, 11(2), 623-634.

Levitin, G. (2003). Optimal allocation of multi-state elements in linear consecutively connected systems 
with vulnerable nodes. European Journal of Operational Research, 150(2), 406- 419.

Lin, H-D., Chen, Y-R., Chiu, V., \& Chiu, Y-S.P. (2019). A decision model for a quality-assured EPQbased intra-supply chain system considering overtime option. Journal of Applied Engineering Science 17(3), 361-371.

Mohammadi, M. (2017). The tradeoff between outsourcing and using more factories in a distributed flow shop system. Economic Computation and Economic Cybernetics Studies and Research, 51(4), 279295.

Morales, F., Franco, C., \& Mendez-Giraldo, G. (2018). Dynamic inventory routing problem: Policies considering network disruptions. International Journal of Industrial Engineering Computations, 9(4), 523-534.

Mosca, A., Vidyarthi, N., \& Satir, A. (2019). Integrated transportation - inventory models: A review. Operations Research Perspectives, 6, Art. No. 100101.

Rahimi, M., \& Fazlollahtabar, H. (2018). Optimization of a closed loop green supply chain using particle swarm and genetic algorithms. Jordan Journal of Mechanical and Industrial Engineering, 12(2), 77 91.

Sarkar, B. (2013). A production-inventory model with probabilistic deterioration in two-echelon supply chain management. Applied Mathematical Modelling, 37(5), 3138-3151.

Siajadi, H., Ibrahim, R.N., \& Lochert, P.B. (2006). Joint economic lot size in distribution system with multiple shipment policy. International Journal of Production Economics, 102(2), 302-316.

Shakoor, M., Abu Jadayil, W., Jaber, N., \& Jaber, S. (2017). Efficiency assessment in emergency department using lean thinking approach. Jordan Journal of Mechanical and Industrial Engineering, 11(2), 97-103.

Souha, B., Soufien, G., \& Mtibaa, A. (2018). Using system generator to design a hardware implementation of a fault-tolerant control of induction motor for electrical vehicle. Journal of Engineering Research, 6(2), 138-154.

Stažnik, A., Babić, D., \& Bajor, I. (2017). Identification and analysis of risks in transport chains. Journal of Applied Engineering Science, 15(1), 61-70.

Swenseth, S.R., \& Olson, D.L. (2016). Trade-offs in lean vs. outsourced supply chains. International Journal of Production Research, 54(13), 4065-4080.

Taft, E.W. (1918). The most economical production lot. Iron Age, 101, 1410-1412.

Zahraee, S.M., Rohani, J.M., \& Wong, K.Y. (2018). Application of computer simulation experiment and response surface methodology for productivity improvement in a continuous production line: Case study. Journal of King Saud University - Engineering Sciences, 30(3), 207-217.

\section{Appendix - A}

Derivation of Eq. (28) is provided as follows:

First, the results of integration for the denominator and numerator of Eq. (27) are exhibited in Eqs. (A1) and (A-2).

$$
\begin{aligned}
& E\left[\boldsymbol{T}_{\pi}\right]=\frac{t_{1 \pi} P_{1} y_{1}}{\lambda}+g\left[1-e^{-\beta t_{1 \pi}}\right] \\
& \left\{\int_{0}^{t_{1 \pi}} E\left[T C\left(t_{1 \pi}\right)_{1}\right] \cdot f(t) d t+\int_{t_{1 \pi}}^{\infty} E\left[T C\left(t_{1 \pi}\right)_{2}\right] \cdot f(t) d t\right\} \\
& =K_{\pi}+K+n K_{1}+t_{1 \pi} \delta_{1}+t_{1 \pi}^{2} \delta_{2}+M\left(1-e^{-\beta t_{1 \pi}}\right)+C_{T} \lambda g\left(1-e^{-\beta t_{1 \pi}}\right)+C_{1} \lambda g\left(1-e^{-\beta t_{1 \pi}}\right) \\
& \quad+h\left(P_{1} g\right)\left(-t_{1 \pi} e^{-\beta t_{1 \pi}}-\frac{1}{\beta} e^{-\beta t_{1 \pi}}+\frac{1}{\beta}\right)+h_{3} \lambda g^{2}\left(1-e^{-\beta t_{1 \pi}}\right)+\frac{1}{2} h_{2} \lambda g^{2}\left(1-e^{-\beta t_{1 \pi}}\right) \\
& \quad+\frac{g}{2 n}\left(h_{2}-h\right)\left(t_{1 \pi} P_{1}\right)\left(y_{1}-y_{2}\right)\left(1-e^{-\beta h_{1 \pi}}\right)+\frac{h g}{2}\left(t_{1 \pi} P_{1}\right)\left(y_{1}-y_{2}\right)\left(1-e^{-\beta t_{1 \pi}}\right) \\
& \quad+\frac{g}{2}\left[h_{2}+2 h_{3}\right]\left(t_{1 \pi} P_{1}\right)\left(y_{1}+y_{2}\right)\left(1-e^{-\beta t_{1 \pi}}\right)
\end{aligned}
$$


where

$$
\begin{aligned}
& \delta_{1}=C_{\pi} \pi\left[\frac{P_{1}}{(1-\pi)}\right]+C P_{1}+C_{T} y_{1} P_{1}+C_{S} E[x] P_{1} \\
& \delta_{2}=\frac{P_{1}^{2}\left(h_{2}-h\right) y_{0}}{2 n \lambda(1-\pi)}\left(y_{1}-y_{2}\right)+\frac{h_{2}}{2} \frac{P_{1} y_{0}}{(1-\pi)}+\frac{h}{2 \lambda} \frac{P_{1}^{2}}{(1-\pi)}\left[\frac{y_{0}^{2}}{(1-\pi)}+\frac{\lambda}{P_{1}}[E[x](1-\pi)-\pi]\right]
\end{aligned}
$$

Then, with additional derivation, $E\left[T C U\left(t_{1 \pi}\right)\right]$ is gained as follows:

$$
E\left[T C U\left(t_{1 \pi}\right)\right]=\left[\frac{\lambda}{y_{1}+\frac{\lambda g\left[1-e^{-\beta t_{1 \pi}}\right]}{t_{1 \pi} P_{1}}}\right]\left(\begin{array}{l}
\frac{v_{0}}{t_{1 \pi}}+\frac{v_{1}}{t_{1 \pi}}+v_{2}+t_{1 \pi} v_{4}-h g e^{-\beta t_{1 \pi}}+\frac{v_{3} e^{-\beta t_{1 \pi}}}{t_{1 \pi}} \\
+v_{5}-v_{5} e^{-\beta t_{1 \pi}}
\end{array}\right)
$$

where

$$
\begin{aligned}
& v_{0}=\frac{K_{\pi}}{P_{1}}+\frac{K}{P_{1}}+\frac{n K_{1}}{P_{1}} \\
& v_{1}=\left[\frac{M}{P_{1}}+\frac{C_{T} \lambda g}{P_{1}}+\frac{C_{1} \lambda g}{P_{1}}+\frac{h_{3} \lambda g^{2}}{P_{1}}+\frac{1}{2} \frac{h_{2} \lambda g^{2}}{P_{1}}+\frac{h g}{\beta}\right] \\
& v_{2}=\left[C_{\pi} \pi\left[\frac{1}{(1-\pi)}\right]+C+C_{T} y_{1}+C_{S} E[x]\right] \\
& v_{3}=\left[-\frac{M}{P_{1}}-\frac{C_{T} \lambda g}{P_{1}}-\frac{C_{1} \lambda g}{P_{1}}-\frac{h_{3} \lambda g^{2}}{P_{1}}-\frac{1}{2} \frac{h_{2} \lambda g^{2}}{P_{1}}-\frac{h g}{\beta}\right] \\
& v_{4}=\frac{P_{1} y_{1}}{2 n \lambda}\left(h_{2}-h\right)\left(y_{1}-y_{2}\right)+\frac{h_{2} P_{1} y_{0} y_{2}}{2 \lambda(1-\pi)}+\frac{h}{2 \lambda}\left[\frac{P_{1}}{(1-\pi)}\right]\left[\frac{y_{0}^{2}}{(1-\pi)}+\frac{\lambda}{P_{1}}[E[x](1-\pi)-\pi]\right] \\
& v_{5}=\frac{h g}{2}\left[\frac{y_{0}}{(1-\pi)}-\frac{\lambda}{P_{1}}\right]+\frac{g}{2 n}\left(h_{2}-h\right)\left(y_{1}-y_{2}\right)+\frac{g}{2}\left(h_{2}+2 h_{3}\right)\left(y_{1}+y_{2}\right)
\end{aligned}
$$

\section{Appendix - B}

The first- and second-derivatives of $E\left[T C U\left(t_{1 \pi}\right)\right]$ are displayed in the following Eqs. (B-1) and (B-2):

$$
\frac{d E\left[T C U\left(t_{1 \pi}\right)\right]}{d\left(t_{1 \pi}\right)}=\frac{\lambda}{\left[y_{1} t_{1 \pi} P_{1}+\lambda g\left(1-e^{-\beta t_{1 \pi}}\right)\right]^{2}}\left\{\begin{array}{l}
-\left(v_{0}+v_{1}\right) P_{1}\left(y_{1} P_{1}+\lambda g \beta e^{-\beta t_{1 \pi}}\right) \\
+v_{3} P_{1}\left(-y_{1} t_{1 \pi} P_{1} \beta e^{-\beta t_{1 \pi}}-\lambda g \beta e^{-\beta t_{1 \pi}}-y_{1} P_{1} e^{-\beta t_{1 \pi}}\right) \\
+v_{4} t_{1 \pi} P_{1}\left(y_{1} t_{1 \pi} P_{1}+2 \lambda g-2 \lambda g e^{-\beta t_{1 \pi}}-t_{1 \pi} \lambda g \beta e^{-\beta t_{1 \pi}}\right) \\
-\left(h g+v_{5}\right) P_{1}\left(\begin{array}{l}
-y_{1} t_{1 \pi}{ }^{2} P_{1} \beta e^{-\beta t_{1 \pi}}-\lambda g e^{-2 \beta t_{1 \pi}} \\
-t_{1 \pi} \lambda g \beta e^{-\beta t_{1 \pi}}+\lambda g e^{-\beta t_{1 \pi}}
\end{array}\right) \\
-\left(v_{2}+v_{5}\right) P_{1} \lambda g\left(t_{1 \pi} \beta e^{-\beta t_{1 \pi}}+e^{-\beta t_{1 \pi}}-1\right)
\end{array}\right\}
$$

and 


$$
\begin{aligned}
& \frac{d^{2} E\left[T C U\left(t_{1 \pi}\right)\right]}{d\left(t_{1 \pi}\right)^{2}}=\frac{\lambda}{\left(y_{1} t_{1 \pi} P_{1}+\lambda g\left(1-e^{-\beta t_{1 \pi}}\right)\right)^{3}} \cdot \\
& \left\{\begin{array}{c}
\left(\begin{array}{l}
\left.v_{0}+v_{1}\right) P_{1}\left(2 y_{1}^{2} P_{1}^{2}+y_{1} t_{1 \pi} P_{1} \lambda g \beta^{2} e^{-\beta h_{1 \pi}}+4 y_{1} P P_{1} \lambda g \beta e^{-\beta t_{1 \pi}}+\lambda^{2} g^{2} \beta^{2} e^{-2 \beta t_{1 \pi}}+\lambda^{2} g^{2} \beta^{2} e^{-\beta t_{1 \pi}}\right.
\end{array}\right) \\
+v_{3} P_{1} e^{-\beta t_{1 \pi}}\left(\begin{array}{l}
y_{1}^{2} t_{1 \pi}{ }^{2} P_{1}^{2} \beta^{2}+2 y_{1}^{2} P_{1}^{2}+2 y_{1}^{2} t_{1 \pi} P_{1}^{2} \beta+2 y_{1} t_{1 \pi} P_{1} \lambda g \beta^{2}+2 y_{1} P_{1} \lambda g \beta \\
+y_{1} t_{1 \pi} P_{1} \lambda g \beta^{2} e^{-\beta t_{1 \pi}}+2 y_{1} P_{1} \lambda g \beta e^{-\beta t_{1 \pi}}+\lambda^{2} g^{2} \beta^{2} e^{-\beta t_{1 \pi}}+\lambda^{2} g^{2} \beta^{2}
\end{array}\right) \\
+v_{4} P_{1} \lambda g\left(\begin{array}{l}
y_{1} t_{1 \pi}{ }^{3} P_{1} \beta^{2} e^{-\beta t_{1 \pi}}+2 \lambda g e^{-2 \beta t_{1 \pi}}+t_{1 \pi}{ }^{2} \lambda g \beta^{2} e^{-2 \beta t_{1 \pi}}-4 \lambda g e^{-\beta t_{1 \pi}} \\
+4 t_{1 \pi} \lambda g \beta e^{-2 \beta t_{1 \pi}}+t_{1 \pi}{ }^{2} \lambda g \beta^{2} e^{-\beta t_{1 \pi}}-4 t_{1 \pi} \lambda g \beta e^{-\beta t_{1 \pi}}+2 \lambda g
\end{array}\right) \\
-\left(h g+v_{5}\right) P_{1} e^{-2 \beta t_{1 \pi}}\left(\begin{array}{l}
y_{1}^{2} t_{1 \pi}{ }^{3} P_{1}^{2} \beta^{2} e^{\beta t_{1 \pi}}+2 y_{1} t_{1 \pi}{ }^{2} P_{1} \lambda g \beta^{2} e^{\beta t_{1 \pi}}-2 y_{1} t_{1 \pi} P_{1} \lambda g \beta e^{\beta t_{1 \pi}} \\
+y_{1} t_{1 \pi}{ }^{2} P_{1} \lambda g \beta^{2}+2 \lambda^{2} g^{2} \beta+4 y_{1} t_{1 \pi} P_{1} \lambda g \beta+t_{1 \pi} \lambda^{2} g^{2} \beta^{2} e^{\beta t_{1 \pi}} \\
+2 y_{1} P_{1} \lambda g-2 y_{1} P_{1} \lambda g e^{\beta t_{1 \pi}}-2 \lambda^{2} g^{2} \beta e^{\beta t_{1 \pi}}+t_{1 \pi} \lambda^{2} g^{2} \beta^{2}
\end{array}\right. \\
+\left(v_{2}+v_{5}\right) P_{1} \lambda g\left(\begin{array}{l}
t_{1 \pi} \lambda g \beta^{2} e^{-2 \beta t_{1 \pi}}+t_{1 \pi} \lambda g \beta^{2} e^{-\beta t_{1 \pi}}+2 \lambda g \beta e^{-2 \beta t_{1 \pi}}-2 \lambda g \beta e^{-\beta t_{1 \pi}} \\
+y_{1} t_{1 \pi}{ }^{2} P_{1} \beta^{2} e^{-\beta t_{1 \pi}}+2 y_{1} P_{1} e^{-\beta t_{1 \pi}}+2 y_{1} t_{1 \pi} P_{1} \beta e^{-\beta t_{1 \pi}}-2 y_{1} P_{1}
\end{array}\right)
\end{array}\right\}
\end{aligned}
$$

Since the first term on the right-hand side (RHS) of Eq. (B-2) is positive, it follows that the $E\left[T C U\left(t_{1 \pi}\right)\right]$ is convex if the second term on the RHS of Eq. (B-2) is also positive. That means if the following $\delta\left(t_{1 \pi}\right)$ $>t_{1 \pi}>0$ holds.

$$
\begin{aligned}
& \left(v_{0}+v_{1}\right)\left(2 y_{1}^{2} P_{1}^{2}+4 y_{1} P_{1} \lambda g \beta e^{-\beta t_{l_{\pi}}}+\lambda^{2} g^{2} \beta^{2} e^{-2 \beta t_{t_{\pi}}}+\lambda^{2} g^{2} \beta^{2} e^{-\beta t_{t_{\pi}}}\right) \\
& +v_{3} e^{-\beta t_{1 \pi}}\left(\begin{array}{l}
2 y_{1}^{2} P_{1}^{2}+2 y_{1} P_{1} \lambda g \beta+\lambda^{2} g^{2} \beta^{2} \\
+2 y_{1} P_{1} \lambda g \beta e^{-\beta t_{1 \pi}}+\lambda^{2} g^{2} \beta^{2} e^{-\beta t_{1 \pi}}
\end{array}\right)+v_{4} \lambda g\left(2 \lambda g e^{-2 \beta t_{1 \pi}}-4 \lambda g e^{-\beta t_{1 \pi}}+2 \lambda g\right)
\end{aligned}
$$

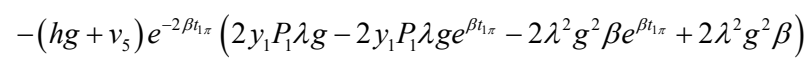

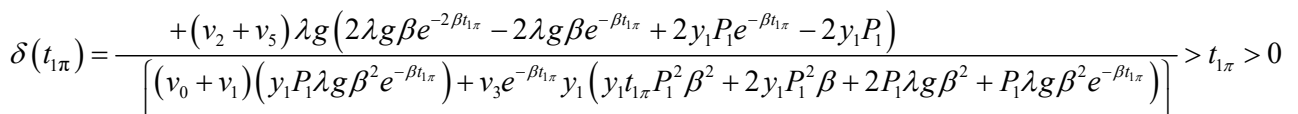

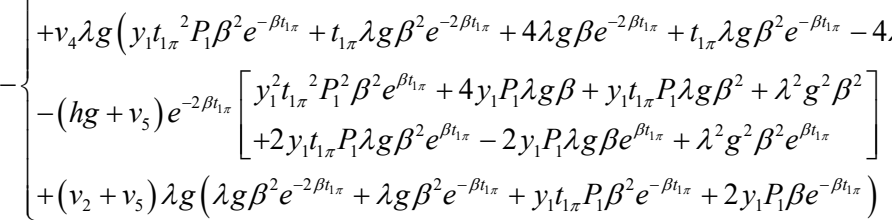

\section{Appendix - C}

\section{Table C-1}

Verification of convexity of $E\left[T C U\left(t_{1} \pi\right)\right]$ against different $\beta S$

\begin{tabular}{ccccc}
\hline$\beta$ & $\delta\left(t_{1 \pi \mathrm{L}}\right)$ & $t_{1 \pi \mathrm{L}}$ & $\delta\left(t_{1 \pi \mathrm{U}}\right)$ & $t_{1 \pi \mathrm{U}}$ \\
\hline 11 & 0.0430 & 0.0199 & 1.0343 & 0.2979 \\
8 & 0.0576 & 0.0265 & 0.6727 & 0.2980 \\
5 & 0.0879 & 0.0393 & 0.4922 & 0.2983 \\
4 & 0.1067 & 0.0467 & 0.4616 & 0.2985 \\
3 & 0.1358 & 0.0570 & 0.4474 & 0.2988 \\
1 & 0.1872 & 0.0720 & 0.4592 & 0.2994 \\
0.5 & $\mathbf{0 . 3 1 3 9}$ & $\mathbf{0 . 0 9 4 0}$ & $\mathbf{0 . 5 4 8 0}$ & $\mathbf{0 . 3 0 1 2}$ \\
0.01 & 0.5266 & 0.1086 & 0.7435 & 0.3047 \\
\hline
\end{tabular}


(C) 2020 by the authors; licensee Growing Science, Canada. This is an open access article distributed under the terms and conditions of the Creative Commons Attribution (CCBY) license (http://creativecommons.org/licenses/by/4.0/). 